\title{
Electron and photon identification in ATLAS.
}

\section{Comparison between test beam data and simulation.}

\author{
F. Derue ${ }^{1}$ - for the ATLAS collaboration \\ LPNHE - Laboratoire de Physique Nucléaire et de Hautes Énergies. IN2P3/CNRS - Universités Paris VI et Paris VII, France
}

Received: date / Revised version: date

\begin{abstract}
The ATLAS experiment at the Large Hadron Collider (LHC) will face the challenge of efficiently selecting interesting candidate events in $p p$ collisions at $14 \mathrm{TeV}$ centre-of-mass energy, whilst rejecting the enormous number of background events. In this talk an overview of the current physics and system performance of the offline selection for electrons and photons is given. Test-beam data, covering a large part of the final detector, have been analysed, and measurements using various important particle identification criteria and methods are presented. The particle identification performance has also been evaluated using detailed Monte Carlo simulations. The efficiency for the signal channels as well as the background rejection capability will be highlighted.
\end{abstract}

PACS. PACS-key ATLAS experiment - PACS-key offline selection of electrons and photons - PACS-key combined test beam results

\section{Introduction}

The CERN Large Hadron Collider (LHC) is a protonproton collider with $14 \mathrm{TeV}$ energy in the centre of mass and a design luminosity of $10^{34} \mathrm{~cm}^{-2} \mathrm{~s}^{-1}$. The ATLAS (A Toroidal LHC ApparatuS) experiment is one of the two major multi-purpose detectors currently under construction at the LHC. Its inner detector consists of tracking detectors enclosed in a solenoidal magnet with $2 \mathrm{~T}$ field. From the inner radius $(5 \mathrm{~cm})$ to the outside radius $(107 \mathrm{~cm})$ it consists of pixel detectors, silicon strip detectors (SCT) and transition radiation drift tubes (TRT), covering the pseudo-rapidity interval $|\eta|<2.5$.

The inner detector is surrounded by a sampling electromagnetic calorimeter based on lead and liquid argon (LAr) technology and a hadronic calorimeter based on LAr in the end-caps and on iron/scintillator tiles in the barrel. The global detector dimensions (diameter $22 \mathrm{~m}$, length $42 \mathrm{~m}$ ) are defined by a large air-core muon spectrometer, providing precision measurements of high- $p_{T}$ muons over $|\eta|<2.5$.

The physics programme envisaged ranges from the search for the Higgs boson, which is the last missing particle within the Standard Model (SM), searches for physics beyond the SM such as supersymmetric particles, new additional $\mathrm{W}$ and $\mathrm{Z}$ bosons and also precision studies, such as measurements of the $t$ quark and $\mathrm{W}$ boson masses and unexpected signals from unpredicted physics scenarios.

\section{The electron and photon selection goals}

Events with electrons and photons in the final state are important signatures for many physics analyses envisaged at the LHC, since electrons and photons are relatively easy to measure precisely and to trigger on. Isolated high- $p_{T}$ electrons and photons are not easy to identify at the LHC because of the very large QCD background from high- $p_{T}$ jets, which result in an electron/jet ratio of about $10^{-5}$ at the LHC (to be compared to about $10^{-3}$ at the Tevatron) for isolated electrons from $W / Z$ decays, and to a photon/jet ratio of about $10^{-4}$ (to be compared to about $10^{-3}$ at the Tevatron). Nevertheless, final states containing several electrons or photons such as $H \rightarrow 4 e$ or $H \rightarrow \gamma \gamma$ decays provide convincing discovery channels [1].

Electron and photon reconstruction mainly exploits data coming from the electromagnetic calorimeter and the Inner Detector (ID) systems. As described in detail in the next sections, electromagnetic objects can be identified in the calorimeter by looking at the transverse and longitudinal shower shapes and at isolation variables. For electrons, a track is then required to match in position and energy that measured in the electromagnetic calorimeter. For photons no track is required (except in the frequent case of converted photons) but $\gamma / \pi^{0}$ separation criteria are required using some of the unique features of the electromagnetic calorimeter. 


\section{Beam test performance}

\section{1 the Transition radiation tracker}

The Transition Radiation Tracker (TRT) is one of the components of the ATLAS Inner Detector. It combines electron identification capability with charged-particle track reconstruction. This is achieved by interleaving layers of xenon-filled drift tubes of small diameter (straws) with radiators. In order to test the physics performance of the proposed detector, several small-scale TRT prototypes were built and tested in the $\mathrm{H} 8$ beam line at the CERN SPS accelerator over the past years. A detailed description of the test beam setup and of the measurement results can be found in [2]

Electron identification makes use of the large energy depositions due to the transition radiation (TR). Typical TR photon energy depositions in the TRT are $8-10 \mathrm{keV}$, while minimum-ionising particles, such as pions, deposit about $1-2 \mathrm{keV}$ (Fig. 1, left).
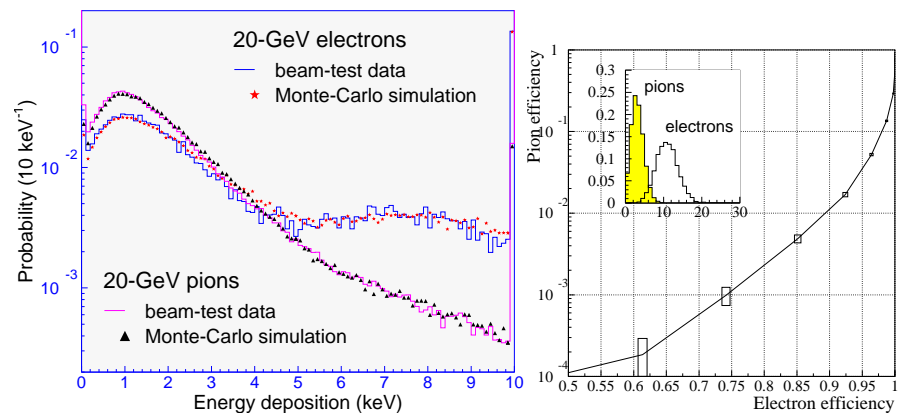

Fig. 1. Differential energy spectra from data and simulation for a single straw with radiator for $20 \mathrm{GeV}$ pions $(\mathrm{dE} / \mathrm{dx})$ and electrons ( $\mathrm{dE} / \mathrm{dx}$ and $\mathrm{TR}$ ) (left); resulting pion efficiency versus electron efficiency at $20 \mathrm{GeV}$ (right) - see [3].

The parameter used in the electron identification is the number of local energy depositions on the track above a given threshold, which whn carried provides a pion versus electron efficiency curve as shown in Fig. 1, right.

The TRT performance was evaluated using electron, pion and muon beams with energies varying from 5 to $300 \mathrm{GeV}$. The distributions of the number of energy depositions for pion and electron tracks reconstructed using a wheel sector prototype are shown for a threshold of $\sim 6$ $\mathrm{keV}$ in the top left-handed corner of Fig. 1 (right). In the same figure, the resulting pion efficiency as a function of the chosen electron efficiency is also displayed. For an electron identification efficiency of $90 \%$, the measured pion efficiency is about $1.2 \%$, i.e a rejection factor of 75 is achieved against $20 \mathrm{GeV}$ pions in a magnetic field of $0.8 \mathrm{~T}$, for a geometry corresponding to that of the ATLAS Inner Detector around $|\eta| \sim 1.2$.

\subsection{The electromagnetic calorimeter}

A detailed description of the barrel and end-cap modules of the ATLAS electromagnetic calorimeter, and of the signal reconstruction techniques can be found in [4]-[5]. Seven production modules, four for the barrel calorimeter (three for the end-cap) were tested in the CERN H8 (H6) beam lines over several months during 2001-2002. The modules reported and in [4]-[5] were pre-production modules ("module 0"s), whereas the results presented here have been obtained with series production modules. Details on the performance obtained with these modules can be found in [6]. The calorimeter performance was measured using secondary or tertiary electron and pion beams, with momenta ranging from 20 to $245 \mathrm{GeV}$ for barrel modules and from 20 to $150 \mathrm{GeV}$ for end-cap modules. The beam lines were equipped with three scintillators in front of the calorimeter for triggering purposes. Four multi-wire proportional chambers allowed to determine the beam impact point at the calorimeter with a resolution of about $250 \mu \mathrm{m}$ in the $\eta$ direction. The size of the last two scintillators, $4 \times 4 \mathrm{~cm}^{2}$, defined the beam acceptance. Cryostats housing the modules were mounted on remotely controlled rails that allowed movements in $\eta$ and $\phi$ while ensuring incident angles similar to the ones expected in ATLAS for all positions. A $3 X_{0}$ lead absorber, a pion counter, a $5 \lambda_{I}$ iron absorber and a muon counter were placed downstream of the cryostat. The readout electronics is similar to the final ATLAS electronics, since it is made of boards functionally identical to the final ones, but, however, do not yet equipped with radiation-resistant ASICs. Energy scans at fixed positions in $\eta$ and $\phi$ were also carried out, and $\eta$-scans were done at fixed electron energy of $245 \mathrm{GeV}$ for the barrel and $120 \mathrm{GeV}$ for the end-cap.
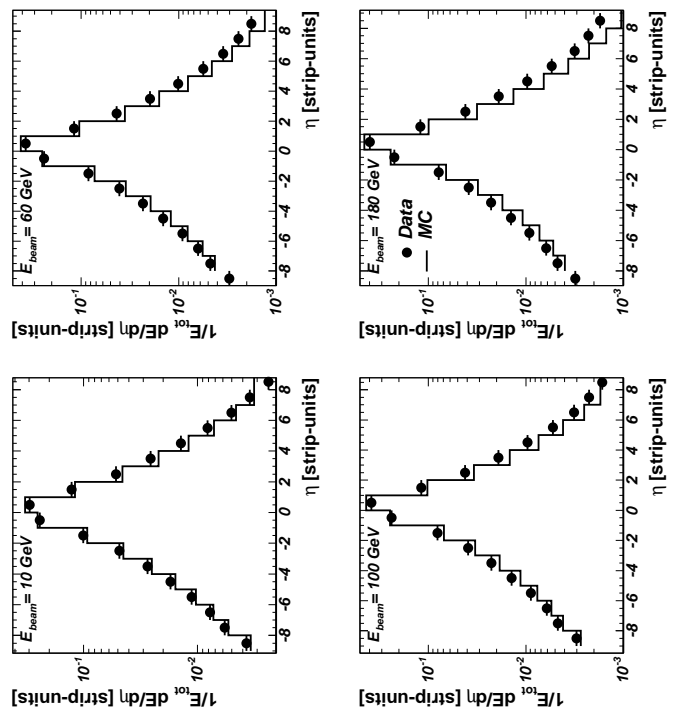

Fig. 2. Lateral shower development of electrons with energies from $10 \mathrm{GeV}$ to $180 \mathrm{GeV}$ in the test beam data (dots) and corresponding G4 simulation (line) - see [8].

Electromagnetic showers initiated by electrons are expected to be essentially contained in the electromagnetic 
calorimeter. The hadronic showers start at a larger depth of the module and there is often a substantial fraction of the total energy shower leaking into the hadronic calorimeter. However, a fraction of hadron-initiated showers may be fully contained creating a potential for particle misidentification. Therefore it is necessary to use the longitudinal and lateral segmentation of the electromagnetic calorimeter to minimise the probability to misidentify hadronic jets as electrons while maintaining high electron identification efficiency.

The data were analysed using the standard ATLAS clustering procedure. The shape of the longitudinal shower profile was contained in the information of the energy $E_{i}$ deposited in each layer of the calorimeter. Additional information was contained in the lateral shower profile, characterised by the number of hit cells in each layer, i.e the number of cells that contain energy well above the noise level. Fig. 2 shows the lateral shower development of electrons in the test beam data and compares them to a G4 standalone simulation. The agreement between data and simulation is good over the energy range from 10 to $180 \mathrm{GeV}$.

In the search for $H \rightarrow \gamma \gamma$ decays, the calorimeter has to provide an additional rejection of about three against $\pi^{0}$ for a photon identification efficiency of $90 \%$, using the fine granularity in the first sampling. This has been demonstrated using specific test-beam data [7], obtained by inserting some material in the beam line upstream of a bending magnet, to cause the incoming electron to emit hard bremsstrahlung photons. By mixing singl photon events with the appropriate kinematics, it was possible to mimick a $\pi^{0}$ decays to two photons. The agreement between simulation and data is satisfactory, and it could be shown that the required rejection factor is reached over most of the kinematical range, as shown in Fig. 3.

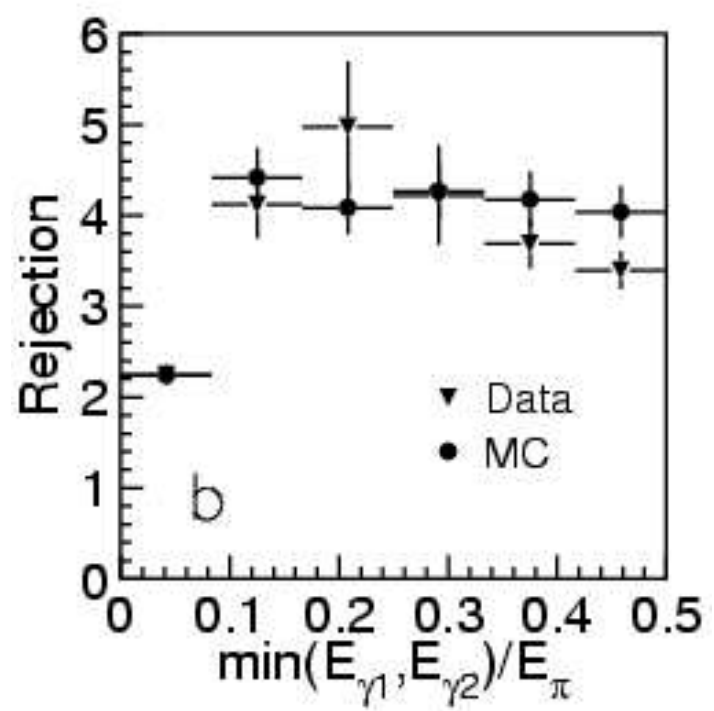

Fig. 3. $\pi^{0}$ rejection calculated in bins of $\min \left(E_{\gamma 1}, E_{\gamma 2}\right) / E_{\pi^{0}}$, for data and simulation [7].

\subsection{The 2004 combined test beam}

In the year 2004, ATLAS has been involved in a huge combined test beam (CTB) effort in the CERN H8 beam line. A complete slice of the barrel detector and of the muon spectrometer end-caps has been tested, with the following clear goals: pre-commission the final elements and study the combined detector performance in a realistic environment, including calibration and alignment. Thanks to this experience, a lot of experience has been acquired in terms of combined operation of all detectors, online monitoring and of data acquisition and triggering, and a considerable amount of data ( 4.6 TB of data, 90 million events) has been collected and is presently under analysis.

A full slice of the ATLAS experiment (Fig. 4) has been tested with beams of different particles (pions, electrons, protons, muons and photons) at different energies and polarities, ranging from $1 \mathrm{GeV}$ up to $350 \mathrm{GeV}$, providing a unique opportunity to evaluate the individual subdetector performances, but also to exploit the full power of the ATLAS detector for detailed particle identification and measurements and to understand better the detector before the commissioning phase. With the data which

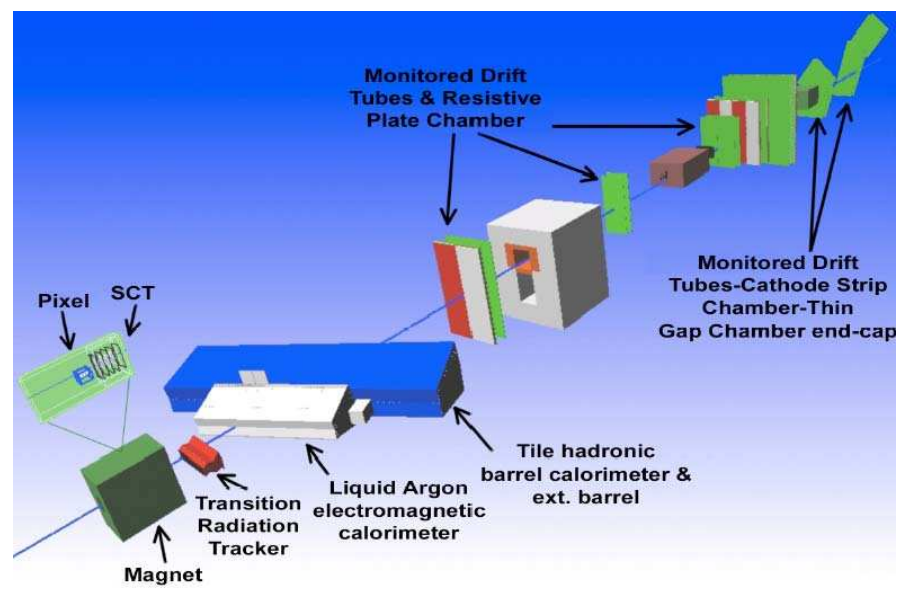

Fig. 4. Geant4 layout of the Combined Test Beam setup.

have been collected, it will be possible to study electron and pion identification and measurement under different conditions (e.g. varying the amount of material in the detector and the magnetic field). Tagged photon beams have also been used to study photon identification and measurement, including in particular photon conversions in the Inner Detector. These data will also be used for detailed G4/FLUKA validation studies and tuning.

\section{Combined ID/EM calorimeter performance}

This section is devoted to a brief discussion of how the combination of the Inner Detector and the EM calorimeter (and to a lesser extent, the hadronic calorimeter) can be used to identify and measure electrons and photons. During the LHC preparation phase, all experiments have 
substantial needs for simulated data in order to estimate the physics performance of the experiment and to prepare the software tools for data analysis. Monte Carlo data are produced during so-called Data Challenges. Most of the results presented in this section is obtained from Data Challenge 1 [9].

\subsection{Photon/jet separation in ATLAS}

Given the amount of material in front of the calorimeter, about $40 \%$ of the photons from e.g $H \rightarrow \gamma \gamma$ decays convert into $e^{+} e^{-}$pairs before depositing their energy in the calorimeter. Since the $H \rightarrow \gamma \gamma$ signal is small, it is important to recover these conversions to maintain its efficiency as high as possible. Conversions at radii below $\sim 80 \mathrm{~cm}$ are reconstructed in the ID. For such events and the energy deposited in the calorimeter in a $3 \times 7(\eta \times \phi)$ window is computed, rather than the standard $3 \times 5$ window used for unconverted photons. The larger window size collects most of the energy of the electron pair and of possible bremsstrahlung photons while preserving excellent energy resolution.

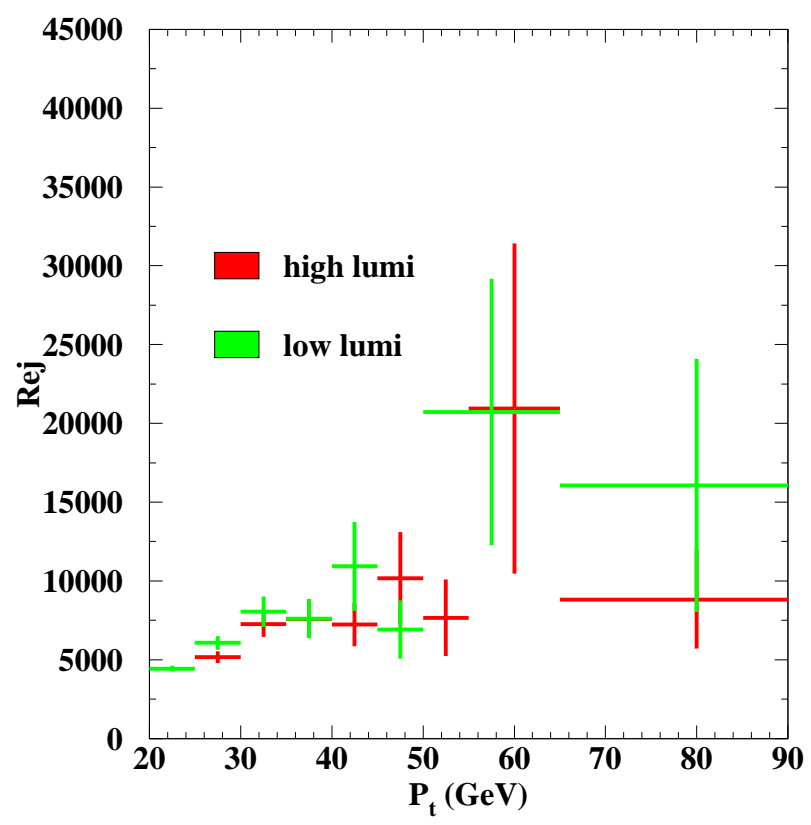

Fig. 5. For a photon identification efficiency of $80 \%$, the jet rejection is obtained as a function of the jet $E_{T}$ for events at low luminosity $\left(2 \times 10^{33} \mathrm{~cm}^{-2} \mathrm{~s}^{-1}\right)$ and high luminosity $\left(10^{34}\right.$ $\left.\mathrm{cm}^{-2} \mathrm{~s}^{-1}\right)[10]$.

In the more general case, the photon/jet separation relies on the search for electromagnetic objects, with cuts which include Level-1 and High Level Trigger cuts, shower shape and isolation cuts in the calorimeter, and the requirement that no track is found in the ID within a $\Delta \eta \times$ $\Delta \phi$ region of size $\pm 0.1 \times \pm 0.1$ around the calorimeter cluster. Fig. 5 shows the jet rejection after photon selection cuts as a function of the jet transverse energy $E_{T}$. A rejection of better than 7000 can be obtained for $E_{T}>40 \mathrm{GeV}$, both for low and high luminosity.

\subsection{Electron/pion separation in ATLAS}

The efficient tagging of low energy electrons is an important tool for B-physics, as well as a complementary method to b-tagging. Separating low energy electrons from pions by analysing the energy deposits in the calorimeter alone is not an easy task, since these electrons are within or near to jets. Instead the ID must be used to seed the calorimeter clustering. The strategy consists of several steps. First, tracks with $p_{T}>2 \mathrm{GeV} / \mathrm{c}$ are found in the ID and then one looks the EM calorimeter regions hit by the tracks. By combining various shower shape estimators, the $E / p$ value and the information from the TRT, it is possible to get the pion rejection versus the electron identification efficiency curves of Fig. 6.

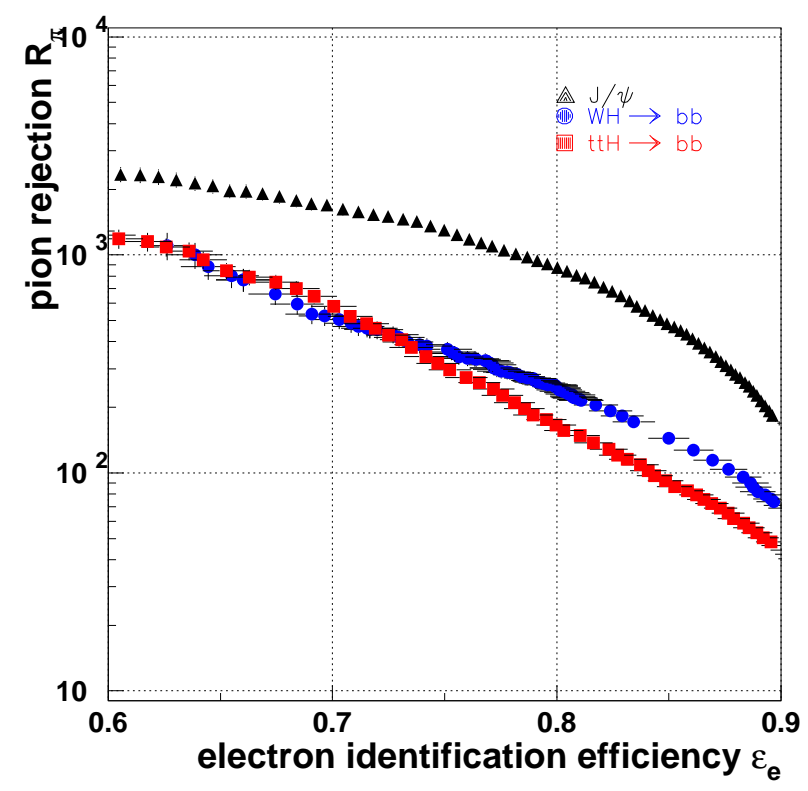

Fig. 6. Pion rejection versus electron identification efficiency for low energy electrons [12]-[13].

In the case of a $J / \psi$ sample, a rejection factor of pion tracks 1000 is achieved for an electron identification efficiency of $80 \%$. This allows the reconstruction of $J \psi$ events with a signal to background ratio around 2 . Electrons coming from $W H \rightarrow b \bar{b}$ events are located inside jets. Thus their identification is harder. For a $80 \%$ identification efficiency, rejection of pion tracks from background sample is $\sim 250$. This soft electron identification could then be used for $\mathrm{b}$ tagging purpose, and has been shown to be a complementary method to standard vertex-based tagging, despite the small branching ratio. 


\subsection{Electron/jet separation in ATLAS}

The identification of isolated electrons with $p_{T}>20 \mathrm{GeV} / \mathrm{c}$ will be essential for the physics searched at the LHC. A challenging task is to identify electrons in the presence of a huge QCD jets background, which is $\sim 10^{5}$ times higher, as in the case of $W$ and top decays.

Table 1. Electron identification efficiency $\varepsilon_{e}$ for single electrons with $p_{T}>25 \mathrm{GeV}$ and jet rejection (with $p_{T}>22 \mathrm{GeV}$ ) of the offline analysis at low luminosity [11].

\begin{tabular}{lll}
\hline & $\varepsilon_{e}($ in $\%)$ & jet rejection $\left(\times 10^{3}\right)$ \\
\hline Calo & $91.2 \pm 0.4$ & $1.3 \pm 0.1$ \\
ID & $81.3 \pm 0.5$ & $19.5 \pm 2.5$ \\
ID-Calo & $76.4 \pm 0.6$ & $57.0 \pm 7$ \\
\hline
\end{tabular}

To separate electrons from jets, cuts were developed to maintain a reasonable electron identification efficiency even with pile-up at high luminosity, while removing a high fraction of jet events. The cuts include Level-1 and High Level Trigger cuts, shower shape and isolation cuts in the calorimeter, cuts on track in ID, cuts on ID-Calo matching in position and energy and transition radiation cuts. The effect of applying all these cuts one after the other to a single elctron sample and an inclusive jet sample are shown in Tab. 1. As in the case of photon/jet separation, after calorimeter selection the dominant background consists of photons from $\pi^{0}$ and $\eta$ decays. This is significantly reduced by requiring the presence of a high $p_{T}$ track. After the ID-Calo match, charged hadron remain as the main background. The signal-to-background ratio is 2:1 for a $Q C D$-jet rejection of $0.6 \times 10^{5}$. The signal is equally from semileptonic decays of heavy quarks and isolated electrons from $W$ and $Z$ decays. The QCD-jet rejection can be improved by using the transition radiation rejection of the TRT as detailed in section 3.1. An electron identification efficiency of about $70 \%$ is obtained while a QCD-jet rejection above $\times 10^{5}$ is achieved. Finally, removal of photon conversions by direct reconstruction, would allow the identification of a pure electron inclusive sample with a jet rejection around $10^{6}$.

\section{Conclusion}

The ATLAS collaboration has devoted considerable effort over the past years to ever more complex test-beam data taking, culminating with the combined test-beam measurements which ended in fall 2004. The analysis of these data, focusing on complex issues such as those related to identification and measurement of electrons in magnetic field and to the reconstruction of photon conversions, will provide strong guidance to tune and validate the software tools needed for ATLAS. This thorough preparation is one of several prerequisite for the delivery of high quality physics data during the initial operation of ATLAS at the LHC. In the meantime, powerful electron and photon identification algorithms were developped and tuned over the past years on detailed Monte Carlo simulation. While maintaining high electron and photon identification efficiency, these algorithms allow to reach very high QCDjet rejection. These strong identification ability coupled with performance of detector themselves will be crucial for many important discovery channels.

\section{References}

1. "ATLAS detector and physics performance technical design report", ATLAS TDR 14 \& 15, CERN/LHCC/99-14 \& 15.

2. T. Akesson et al. (ATLAS Coll.), "ATLAS Transition Radiation Tracker test-beam results", Nucl. Instrum. Meth. A522, (2004) 50-55.

3. V. Mitsou et al. (ATLAS Coll.), "The ATLAS Transition Radiation Tracker", ATL-CONF-2003-012 (2003), proc. of ICATPP 2003

4. B. Aubert et al. (ATLAS Coll.), "Performance of the ATLAS electromagnetic calorimeter end-cap module 0", Nucl. Instrum. Meth. A500, (2003) 178-201.

5. B. Aubert et al. (ATLAS Coll.), "Performance of the ATLAS electromagnetic calorimeter end-cap module 0", Nucl. Instrum. Meth. A500, (2003) 202-231.

6. Ph. Schwemling et al. (ATLAS Coll.), "ATLAS electromagnetic calorimetry and performance of electron/photon detection", Eur. Phys. J., C 34 (2004)

7. J. Colas et al. (ATLAS EMLarg Coll.), "Position resolution and particle identification with the ATLAS EM calorimeter", Nucl. Instrum. Meth. A550, (2005) 96-115

8. T. Carli et al. (ATLAS EMLarg Coll.), "Energy linearity and resolution performance of the ATLAS electromagnetic barrel calorimeter in the CERN electron beamr", to be submitted to NIM.

9. R. Sturrock et al. (ATLAS Coll.), "A Step Towards A Computing Grid For The LHC Experiments : ATLAS Data Challenge 1", Nucl. Instrum. Meth. A502, (2003) 446-449

10. M. Escalier et al., "Photon/jet separation with DC1 data", ATLAS Note ATL-COM-PHYS-2005-048

11. F. Derue, C. serfon "Electron/jet separation with DC1 data", ATLAS Note ATL-PHYS-PUB-2005-016

12. T. Bold et al., "Pile-up studies for soft electron identification and b-tagging with DC1 data", ATLAS Note ATLCOM-PHYS-2005-027

13. F. Derue et al., "Reconstruction of DC1 $J / \psi \rightarrow e^{+} e^{-}$ decays and use for the low energy calibration of the ATLAS electromagnetic calorimeter", ATLAS Note ATL-COMPHYS-2005-022 